\title{
Clinical benefits of diffusion tensor imaging in hydrocephalus
}

\author{
*Liat Ben-Sira, MD, ${ }^{1}$ Noam Goder, MD, ${ }^{1}$ Haim Bassan, MD, ${ }^{2}$ Shlomi Lifshits, MSc, ${ }^{3}$ \\ Yaniv Assaf, PhD, ${ }^{4}$ and Shlomi Constantini, MD, MSc ${ }^{5}$
}

\begin{abstract}
1Pediatric Neuroimaging Unit, ${ }^{2}$ Pediatric Neurology and Development, and ${ }^{5}$ Department of Pediatric Neurosurgery, Tel Aviv Sourasky Medical Center, The Sackler Faculty of Medicine; ${ }^{3}$ School of Statistics and Operational Research, The Sackler Faculty of Exact Sciences; ${ }^{4}$ Department of Neurobiology, The George S. Wise Faculty of Life Sciences, Tel Aviv University, Tel Aviv, Israel
\end{abstract}

OBJECT The object of this study was to use diffusion tensor imaging (DTI) to evaluate and characterize white matter changes in hydrocephalus.

METHODS The authors performed a retrospective analysis of DTI in a cohort of patients with hydrocephalus $(n=35)$, 19 of whom had both pre- and postsurgical imaging studies. These patient's DTI values were compared with values extracted from age-dependent trend lines computed from a healthy subject group ( $n=70$, age span 14 months-14 years). Several DTI parameters in different regions of interest (ROIs) were evaluated to find the most sensitive parameters for clinical decision making in hydrocephalus.

RESULTS Compared with healthy controls, patients with active hydrocephalus had a statistically significant change in all DTI parameters. The most sensitive and specific DTI parameter for predicting hydrocephalus was axial diffusivity $\left(\lambda_{1}\right)$ measured at the level of the corona radiata. Diffusion tensor imaging parameters correlated with several conventional radiological parameters in the assessment of hydrocephalus but were not superior to them. There was no convincing correlation between clinical disease severity and DTI parameters. When examining the pre- and postsurgical effect, it was found that DTI may be a sensitive tool for estimating tissue improvement.

CONCLUSIONS This large-cohort study with a multidisciplinary approach combining clinical, neurological, radiological, and multiple DTI parameters revealed the most sensitive DTI parameters for identifying hydrocephalus and suggested that they may serve as an important tool for the disorder's quantitative radiological assessment.

http://thejns.org/doi/abs/10.3171/2014.10.PEDS13668

KEY WORDS magnetic resonance imaging; diffusion tensor imaging; diffusion magnetic resonance imaging; hydrocephalus; endoscopic third ventriculostomy

$\mathrm{H}$ YDROCEPHALUS is a disorder representing a mismatch between cerebrospinal fluid (CSF) production and absorption and/or an interruption in CSF pathways. In progressive cases, surgery is indicated to prevent damage to brain parenchyma. Occasionally, clinical signs and symptoms are obvious and the indication for surgery is not in question. At times, however, imaging may demonstrate enlarged ventricles, whereas the clinical picture is less obvious and symptoms and signs are subtle.
The clinical dilemma, in such cases, is whether ventriculomegaly per se should be treated or simply monitored. These cases pose a difficult clinical challenge: to operate or not to operate? Current decision making is based almost solely on clinical experience and instincts rather than a validated quantitative paradigm.

The treatment of choice for most cases of hydrocephalus is fluid diversion via a ventriculoperitoneal shunt (VPS). In the last few years, an alternative procedure, endoscopic

ABBREVIATIONS CR = corona radiata; CSF = cerebrospinal fluid; DTI = diffusion tensor imaging; ETV = endoscopic third ventriculostomy; FA = fractional anisotropy; GENU = genu of the corpus callosum; HCP group = hydrocephalus group; ICP = intracranial pressure; $\mathrm{MD}=$ mean diffusivity; $\mathrm{OR}=$ optic radiation; ROI = region of interest; VPS = ventriculoperitoneal shunt; $\lambda_{1}=$ diffusivity parallel to the fibers; $\lambda_{3}=$ diffusivity perpendicular to the fibers.

SUBMITTED December 16, 2013. ACCEPTED October 21, 2014.

INCLUDE WHEN CITING Published online May 15, 2015; DOI: 10.3171/2014.10.PEDS13668.

DISCLOSURE The authors report no conflict of interest concerning the materials or methods used in this study or the findings specified in this paper.

* Drs. Ben-Sira and Goder contributed equally to this work. 
third ventriculostomy (ETV), has become preferred for patients with an obstructive (noncommunicating) hydrocephalus. A VPS diverts the CSF to the peritoneal compartment; therefore, the ventricles commonly become smaller following surgery. In ETV, the diversion occurs within the skull, and after a successful procedure, the size of the ventricles does not significantly change in many patients. Thus, it is difficult to evaluate success after this type of operation. ${ }^{1,6,9}$ Complicating the paradigm in these situations is the fact that, as of today, there is no reliable noninvasive method to assess intracranial pressure (ICP) and to demonstrate the pressure of the fluid on adjacent brain tissue.

Diffusion tensor imaging (DTI) is an advanced MRI technique that characterizes white matter by quantifying the 3D diffusion properties of water in tissue. In white matter, diffusion is anisotropic, meaning that it is free along the path of the fibers and hindered perpendicular to them. Structural components, such as axonal membranes and myelin, significantly influence the anisotropic diffusion of water. Therefore, alterations in DTI parameters are believed to reflect the level of structural integrity of tissue..$^{3,410-12}$ Diffusion tensor imaging extracts the principal diffusivities parallel (represented by $\lambda_{1}$ ) and perpendicular (represented by $\lambda_{2}$ and $\lambda_{3}$ ) to the fibers, the mean diffusivity (MD), and the fractional anisotropy (FA) - a measure of the difference between each of the principal diffusivities and their mean. ${ }^{11}$

Initial experience with the changes in DTI parameters in hydrocephalus was previously presented in a case-control study of 7 patients with hydrocephalus. ${ }^{2}$ That study showed increased FA and $\lambda_{1}$ in the corona radiata (CR) in patients with hydrocephalus before surgery. Several other studies have looked at DTI changes following surgery for hydrocephalus. ${ }^{1,6,7,9,15}$

In the present study, we investigated a group of pediatric patients with ventriculomegaly with various clinical presentations. We evaluated several DTI parameters in different regions of interest (ROIs) to find the most sensitive parameters for clinical decision making in hydrocephalus. We sought to find the relationships among various DTI, clinical, and conventional radiological parameters.

\section{Methods}

\section{Study Design and Patients}

In this retrospective study, which was approved by the Tel Aviv Sourasky Medical Center Helsinki committee, we performed a search of the entire MRI database for all DTI studies obtained between the years 2003 and 2009 by using the terms "hydrocephalus" and "ventriculomegaly." It should be noted that DTI was not a routine protocol and was performed only in particular cases in which the radiologist thought it would be beneficial. We included in our study children 1-14 years of age. Diffusion tensor imaging studies had been obtained during routine MRI sessions performed for various medical indications. We categorized all MR images into 2 groups: the hydrocephalus (HCP) group and the healthy control group. For both groups, we reviewed all medical records and abstracted the relevant demographic, clinical, and surgical information. Patients with MRI findings consistent with hydrocephalus were included in the HCP group based on radiological and clinical assessments made by a pediatric radiologist, pediatric neurologist, and neurosurgeon. The control group consisted of subjects who had normal MRI results or findings that had no known effects on brain white matter (Table 1). These subjects were included in the control group when their clinical records suggested no neurological disorder or developmental delay, as judged by a pediatric neurologist. A subset of the HCP group had DTI studies available from both before and after the surgical intervention for hydrocephalus (pre- and postsurgical scans had been obtained using the same scanner with the same protocol).

\section{Imaging Acquisition}

Magnetic resonance imaging had been performed on a 1.5- or 3-T Signa scanner (GE Healthcare) with a conventional head coil. The field of view in all MRI studies was $24 \mathrm{~cm}$, and the slice thickness was $3 \mathrm{~mm}$ with no gap between slices. In addition to several clinical sequences, the MRI protocol included a T2-weighted series (fast spinecho pulse sequence with TR $5000 \mathrm{msec}$, TE $102 \mathrm{msec}$ ) and a DTI protocol. The DTI data set was acquired using spin-echo echo-planar diffusion-weighted imaging sequences with the following parameters: TR $10,000 \mathrm{msec}$; TE $98 \mathrm{msec} ; \Delta 31 \mathrm{msec}, \delta 25 \mathrm{msec}$, and maximum gradient strength $\left(\mathrm{G}_{\max }\right)$ of 2.2 gauss $/ \mathrm{cm}$ for the 1.5-T scanner and $\Delta 24 \mathrm{msec}, \delta 18 \mathrm{msec}$, and $\mathrm{G}_{\max }$ of 3.4 gauss $/ \mathrm{cm}$ for the 3 -T scanner; matrix $128 \times 128$ with 3 averages. The diffusion-weighted images were acquired along 6 noncollinear diffusion gradient directions with a maximal $b$ value of $1000 \mathrm{~s} / \mathrm{mm}^{2}$. The DTI data set consisted of 7 images $(6$ diffusion-weighted images and 1 image with no applied diffusion gradients). Total acquisition time for the entire DTI data set was 4 minutes.

\section{Neurological and Radiological Assessment \\ Clinico-Neurological Hydrocephalus Severity Grading}

To rate the clinical severity of hydrocephalus we generated a list of clinical signs and symptoms of increased intracranial pressure (ICP) that we commonly see in our clinical practice. We categorized all of these clinical items into 3 disease severity grades: mild, moderate, and severe (Table 2). Then we assigned a clinico-neurological hydrocephalus grade based on the presence of 1 or more items

TABLE 1. Indications for MRI in 70 healthy control subjects

\begin{tabular}{cl}
\hline No. of Subjects & \multicolumn{1}{c}{ Indication } \\
\hline 6 & Extracranial findings \\
\hline 8 & Temporary neurological findings \\
\hline 6 & Abnormal development w/ later catch-up \\
\hline 9 & Suspected intracranial process/headache \\
\hline 17 & Suspected pituitary abnormality \\
\hline 11 & Large head circumference \\
\hline 5 & Seizures \\
\hline 6 & Torticollis, strabismus, Horner's syndrome, ptosis \\
\hline
\end{tabular}


TABLE 2. Clinico-neurological hydrocephalus severity grading

\begin{tabular}{llll}
\hline \multicolumn{1}{c}{ Grade } & \multicolumn{1}{c}{ Infant } & \multicolumn{1}{c}{ Child } & \multicolumn{1}{c}{ Both } \\
\hline Mild & $\begin{array}{c}\text { Increased head } \\
\text { circumference }\end{array}$ & Headaches & Developmental delays \\
\cline { 2 - 4 } & & Nausea & New-onset seizures \\
\cline { 2 - 4 } & & New head tilt \\
\hline Moderate & Sun set eyes & Pyramidal signs \\
\cline { 2 - 4 } & Irritability & Diplopia or EOM palsy \\
\cline { 2 - 4 } & & Vomiting \\
\hline Severe & Bulging fontanelle & Swollen discs & Drowsiness/apathy \\
\cline { 2 - 4 } & & & Bradycardia \\
\cline { 2 - 4 } & & Apnea \\
\hline
\end{tabular}

$\mathrm{EOM}=$ extraocular muscle.

of the most severe clinical grade. For example, headaches and nausea were scored as mild disease; headaches and vomiting as moderate; and headaches and swollen discs as severe. An overall grade was always given after consensus between the study neurosurgeon and neurologist was reached.

\section{Conventional Radiology Evaluation}

Independent researchers blinded to the clinical hydrocephalus severity data obtained the radiological data. The MRI parameters used to assess hydrocephalus severity included the lateral ventricular span, the average of the frontal and occipital horn width divided by the interparietal distance (a parameter found to correlate well with ventricular volume), ${ }^{8}$ third ventricle floor flattening, CSF flow through the aqueduct, herniation of the cerebellar tonsils, fluid in the sleeves around the optic nerves, flattening of the pituitary gland, and periventricular edema. The radiologist was also asked to perform an overall evaluation of the likelihood of increased ICP based on these conventional MRI parameters (yes/no).

\section{Data Analysis}

After selecting the HCP and control groups, we extracted the DTI parameters from each scan. Three ROIs were selected according to the same method used in Assaf et al.: corona radiata (CR), genu of the corpus callosum (GENU), and optic radiation (OR; Fig. 1). In each ROI, the following DTI parameters were extracted: fractional anisotropy (FA), mean diffusivity (MD), diffusivity parallel to the fibers $\left(\lambda_{1}\right)$, and diffusivity perpendicular to the fibers $\left(\lambda_{3}\right)$.

Since DTI parameters vary greatly with age, our study required standardization of the data. Initially, for each DTI parameter in each ROI, the control group values were plotted on a chart and an exponential trend line was fitted. This trend line could predict the expected value for any healthy subject as a function of the subject's age. For these trend lines, the $95 \%$ confidence intervals were computed.

Using the trend line functions, expected DTI index values were computed for each hydrocephalus patient according to his or her age at the time of scanning. This expected value was subtracted from the measured value, and the difference was recorded for all of the DTI parameters for each patient. The average of all of these values (expected value and the difference $[\Delta]$ ) was calculated for each DTI parameter (for example, $\Delta \mathrm{FA} \mathrm{I}_{\mathrm{IC}}$ means the difference in FA in the internal capsule). A paired t-test (comparison between each measured and expected value) was used to check for statistical significance with a threshold of $p<0.05$. For each DTI parameter, sensitivity for $95 \%$ specificity was calculated to assess the ability of these parameters to identify hydrocephalus. Both sensitivity and specificity were calculated against the $95 \%$ confidence interval criterion of the control group. The subjects (from either the HCP or the control group) whose values fell within this confidence interval were marked as "healthy" and the others as "diseased." Based on the numbers of healthy and diseased subjects, specificity and sensitivity were calculated.

For patients who had DTI studies from both before and after surgical intervention, the differences from healthy values were calculated before and after surgery. Then the difference after surgery was subtracted from the difference before surgery to compute a new value, that is, "the difference between differences" ( $\Delta \Delta$; Table 3$)$. For example, $\Delta \Delta F A_{\text {IC }}$ provides the effect of surgery corrected for the different age of the subject at the time of scanning. Again, a paired t-test was used to check for statistical significance with a threshold of $\mathrm{p}<0.05$.

Statistical analysis was performed using Microsoft Excel (Microsoft Corp.) and SPSS version 17 (SPSS Inc.). Multiple linear regressions were made using R, an opensource statistical language.

\section{Results}

Seventy healthy subjects, ages 14 months-14 years, were included in this study following screening (Table 2). Thirty-five patients, ages 1 month-14 years, were included in the HCP group, and 19 of them had both pre- and postsurgical DTI studies. Ten of these surgically treated patients underwent ETV; 3, VPS surgery; and 6, resection of a mass causing hydrocephalus. Of the 16 remaining patients with hydrocephalus, 10 underwent a surgical intervention but did not have postsurgical DTI studies. Four patients did not undergo surgery, and 2 patients were lost to follow-up (Table 4).

According to the clinico-neurological hydrocephalus severity grades, 6 patients had severe hydrocephalus; 16 , moderate hydrocephalus; and 12, mild hydrocephalus. One patient could not be classified because of insufficient clinical data.

For all DTI parameters, the patients with hydrocephalus had values statistically different from those of the healthy subjects (calculated using the difference from the expected value $[\Delta], p<0.05)$. An increase was noted in the average of the following parameters: FA, MD, and $\lambda_{1}$ for the CR and in MD, $\lambda_{1}$ and $\lambda_{3}$ for the GENU and OR. A decrease was noted in $\lambda_{3}$ for the CR and in FA for the GENU and OR. For each DTI parameter, the sensitivity for hydrocephalus at $95 \%$ specificity was calculated (Fig. 2 left and Table 5). The most sensitive DTI parameter for the characterization of hydrocephalus was $\lambda_{1}$ for the CR, in which $89 \%$ sensitivity for $95 \%$ specificity was reached (Fig. 2 right). 

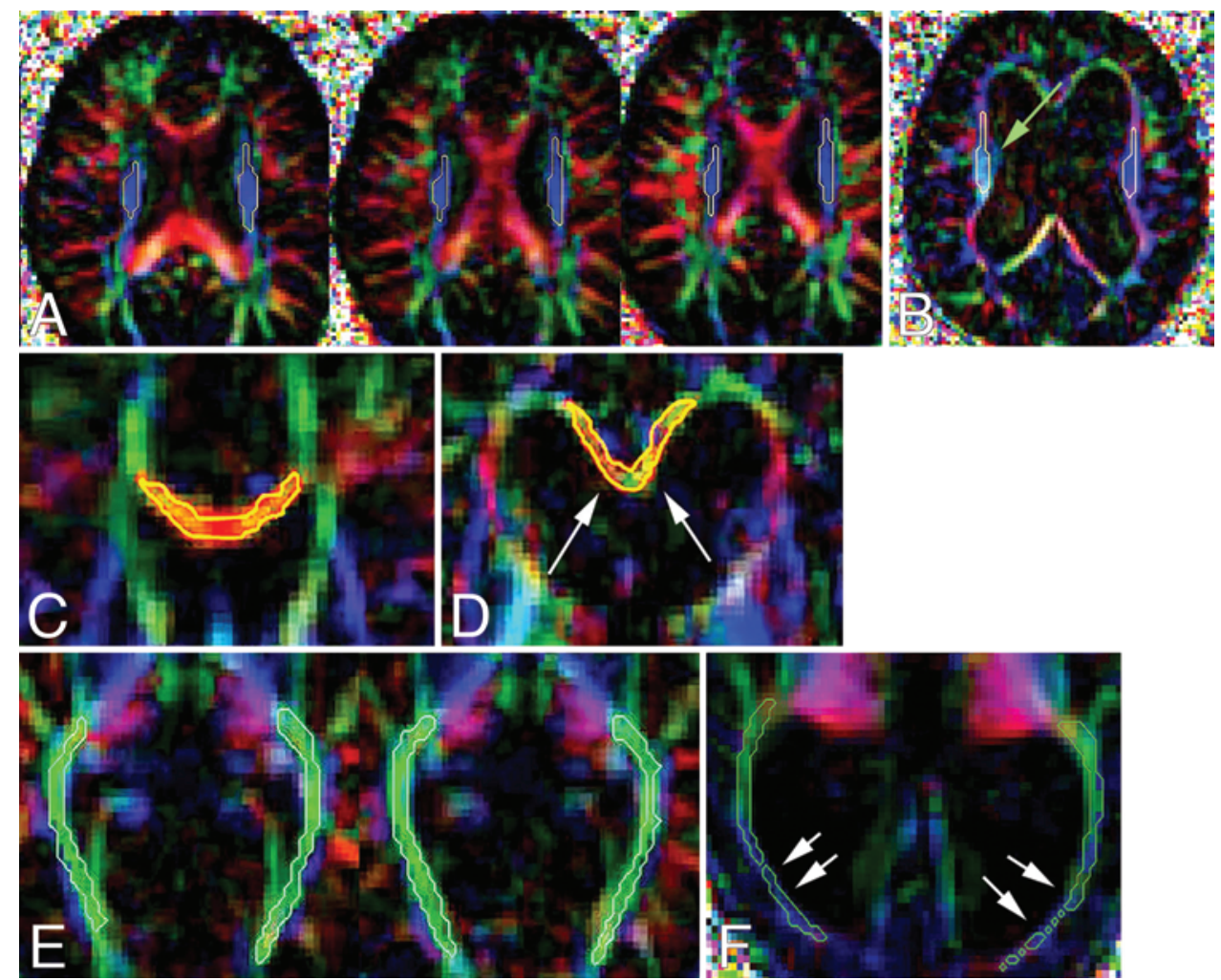

FIG. 1. In the CR, the DTI parameters were extracted from 3 consecutive axial images. Three axial images from a healthy subject (A) and 1 axial image (of 3 obtained) from a patient with hydrocephalus (B). Green arrow indicates an area of signal intensification. In the GENU, the ROI in the axial image with the highest $\lambda_{1}$ intensity was selected-here in a healthy subject (C) and a patient with hydrocephalus (D). In the OR, 2 axial images in which the thalamus was visible were selected-here in a healthy subject (E) and a patient with hydrocephalus (F). In all panels in this figure, left to right fibers are red, anterior to posterior fibers are green, and upward to downward fibers are blue. White arrows in all panels indicate areas of signal attenuation in a patient with hydrocephalus. Figure is available in color online only.

\section{Correlation Between DTI Parameters and Disease Severity}

When the patients with hydrocephalus were classified into 3 clinical groups (mild, moderate, and severe hydrocephalus), we noted a trend toward larger differences from the expected value by age $(\Delta)$ in the severe hydrocephalus subgroup (data not shown). However, no statistically significant difference was found when comparing the 12 patients who were classified as having mild hydrocephalus and the 6 patients who were classified as having severe hydrocephalus. When combining the mild and moderate hydrocephalus subgroups and comparing their differences to those of the severe hydrocephalus subgroup, 1 DTI parameter reached statistical significance. In this setting, $\Delta \lambda_{1}$ for the corpus callosum was $0.35 \times 10^{-3} \mathrm{~mm}^{2} / \mathrm{sec}$ in the severe subgroup (6 patients) and $0.12 \times 10^{-3} \mathrm{~mm}^{2} / \mathrm{s}$ in the combined mild and moderate patient subgroup (28 pa- tients; $p=0.008)$. However, when applying the conservative Bonferroni correction for multiple comparisons, the result did not reach a significant level.

\section{Correlation Between DTI Parameters and Conventional Radiology Parameters}

The greatest correlation between DTI parameters and conventional radiology parameters was found between $\Delta \lambda_{1 C R}$ and the average of the frontal and occipital horn width divided by the interparietal distance, which represents the ventricular volume ${ }^{8}(r=0.687, p<0.01$; Fig. 3). Another parameter found to have the greatest correlation with the size of the ventricles was $\mathrm{MD}_{\mathrm{GENU}}(\mathrm{r}=0.643$, $\mathrm{p}<$ $0.01)$. None of the DTI parameters was highly correlated with the overall radiological evaluation of hydrocephalus severity.

TABLE 3. Example of calculations made for each patient, ROI, and DTI parameter

\begin{tabular}{lcccc}
\hline \multicolumn{1}{c}{ Case 1, ROI: IC } & Age $(\mathrm{yrs})$ & $\mathrm{MD}\left(\times 10^{-3} \mathrm{~mm}^{2} / \mathrm{sec}\right)$ & $\begin{array}{c}\text { Expected MD, Healthy } \\
\text { Value }\left(\times 10^{-3} \mathrm{~mm}^{2} / \mathrm{sec}\right)\end{array}$ & $\begin{array}{c}\text { Difference Btwn Measured } \\
\text { \& Expected }\left(\Delta \mathrm{MD} \mathrm{D}_{\mathrm{IC}}\right)\end{array}$ \\
\hline Before surgery & 0.75 & 1.58 & 1.29 & 0.29 \\
\hline After surgery & 2.17 & 1.39 & 1.24 & 0.15 \\
\hline Difference btwn the differences $\left(\Delta \Delta \mathrm{MD}_{\mathrm{IC}}\right)$ & & & & 0.14 \\
\hline
\end{tabular}

IC = internal capsule. 
TABLE 4. Summary of data for 35 patients with hydrocephalus

\begin{tabular}{|c|c|c|c|c|}
\hline $\begin{array}{l}\text { Case } \\
\text { No. }\end{array}$ & $\begin{array}{c}\text { Age at Imaging Before } \\
\text { Surgery (yrs) }\end{array}$ & $\begin{array}{c}\text { Age at Imaging } \\
\text { After Surgery (yrs) }\end{array}$ & Presumed Etiology & Surgical Intervention \\
\hline 1 & 0.08 & 1.00 & Chiari malformation II & ETV, septum pellucidotomy \\
\hline 2 & 0.67 & 1.92 & Infracerebellar cyst & Marsupialization \\
\hline 3 & 0.75 & 2.17 & Aqueductal stenosis & Shunt \\
\hline 4 & 0.75 & 1.50 & Infracerebellar cyst & ETV \\
\hline 5 & 0.83 & 1.50 & Infracerebellar cyst & ETV \\
\hline 6 & 0.83 & 1.00 & Postbleeding, postinfection & Shunt \\
\hline 7 & 0.83 & 1.00 & Postbleeding & ETV \\
\hline 8 & 1.00 & 1.10 & Posterior fossa tumor & Resection \\
\hline 9 & 1.80 & 2.20 & Choroid plexus tumor & Resection \\
\hline 10 & 2.83 & 3.42 & Postbleeding, infracerebellar cyst & ETV \\
\hline 11 & 3.30 & 4.83 & Aqueductal stenosis & ETV \\
\hline 12 & 4.00 & 5.00 & Posterior fossa tumor & Resection \\
\hline 13 & 4.92 & 5.25 & Postinfection aqueductal stenosis & ETV \\
\hline 14 & 6.50 & 6.67 & Aqueductal stenosis & ETV \\
\hline 15 & 8.00 & 9.00 & Dandy-Walker & Posterior fossa decompression \\
\hline 16 & 12.00 & 12.00 & Foramen of Monro blockage & Endoscopic septum pellucidotomy \\
\hline 17 & 12.00 & 14.00 & Aqueductal stenosis, postinfection & ETV \\
\hline 18 & 13.10 & 13.10 & Dandy-Walker & Shunt revision \\
\hline 19 & 14.00 & 14.00 & Aqueductal stenosis & ETV \\
\hline 20 & 0.13 & & Chiari malformation I & Endoscopic septum pellucidotomy \\
\hline 21 & 0.20 & & Postbleeding aqueductal stenosis & ETV \\
\hline 22 & 0.25 & & Postbleeding aqueductal stenosis & Shunt \\
\hline 23 & 0.25 & & Choroid plexus tumor & Operated on at another hospital \\
\hline 24 & 0.25 & & Infracerebellar cyst & Shunt \\
\hline 25 & 0.83 & & Aqueductal stenosis & ETV \\
\hline 26 & 1.00 & & Chiari malformation II & Was not operated on \\
\hline 27 & 1.00 & & Prematurity & Unknown; lost to follow-up \\
\hline 28 & 1.00 & & Postbleeding & Shunt \\
\hline 29 & 1.00 & & Postbleeding & Posterior fossa decompression \\
\hline 30 & 1.08 & & Chiari malformation I & Unknown; lost to follow-up \\
\hline 31 & 1.25 & & Infracerebellar cyst & Was not operated on \\
\hline 32 & 1.42 & & Chiari malformation I & Was not operated on \\
\hline 33 & 3.50 & & Postbleeding & Was not operated on \\
\hline 34 & 5.50 & & Posterior fossa tumor & Resection \\
\hline 35 & 7.42 & & Tectal tumor & Resection \\
\hline
\end{tabular}

\section{Correlation Among DTI Parameters, Disease Severity, and Conventional Radiology Parameters}

The multiple linear regression analysis between the radiological parameters (measured from conventional MRI) and $\lambda_{1}$ for the CR with disease severity as the dependent variable resulted in an $\mathrm{R}^{2}=0.662$. A similar model excluding $\lambda_{1}$ for the CR (that is, without the use of any DTI parameter) resulted in an $\mathrm{R}^{2}=0.659$. Comparison between these 2 models was made with the F test and was found to be statistically insignificant, with $p=0.66$. These results mean that the clinical assessment of hydrocephalus is not improved by the addition of $\lambda_{1}$ for the CR as the covariate.
The best correlations between disease severity and the various conventional radiological parameters were periventricular edema $(r=0.660, p<0.01)$, overall radiological evaluation $(\mathrm{r}=0.467, \mathrm{p}<0.05)$, and pituitary flattening $(\mathrm{r}$ $=0.435, \mathrm{p}<0.01)$.

\section{Comparison Between DTI Before and After Surgery}

For patients who had an additional DTI study following surgical intervention, we calculated the difference between the differences from the expected healthy trend line $(\Delta \Delta)$ before and after an intervention (Table 3). Overall, 19 patients had DTI studies before and after surgery, 

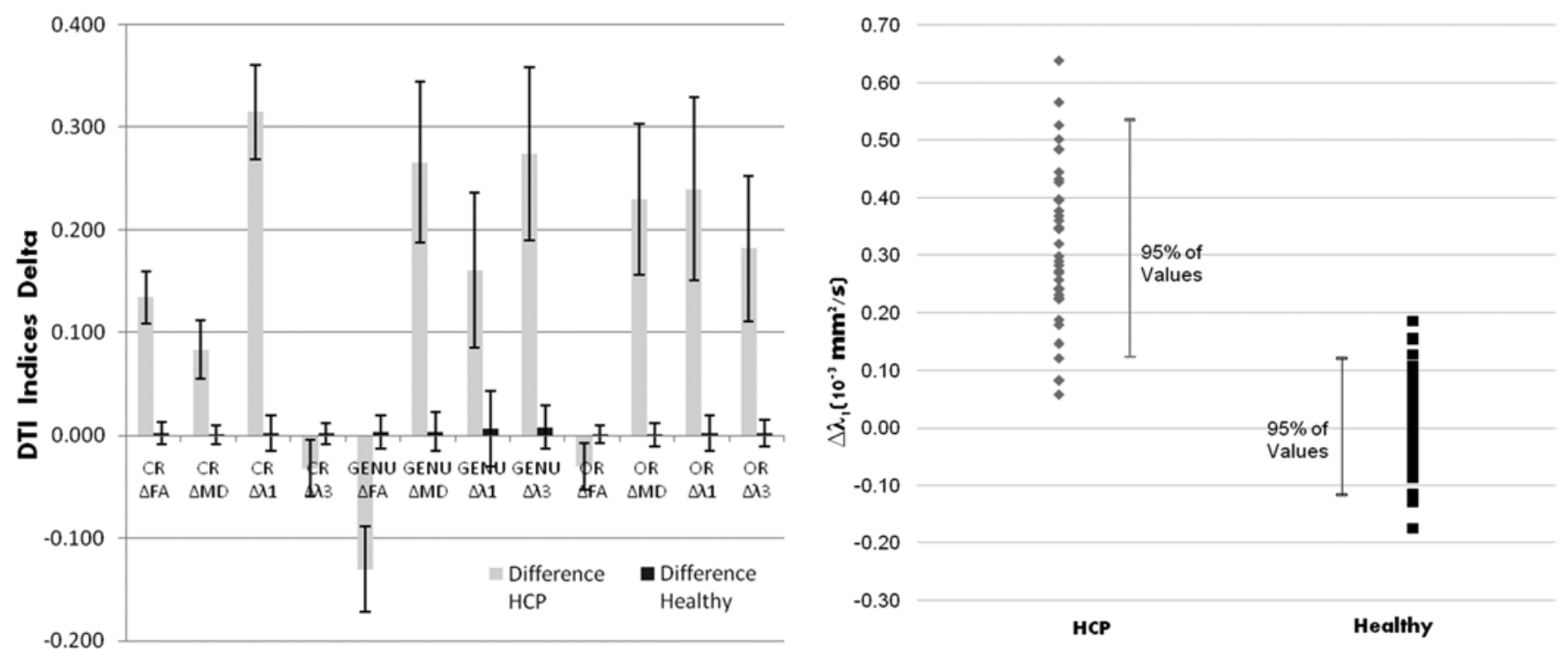

FIG. 2. Left: Average of the differences between the measured DTI parameters and the healthy values expected by age along with the $95 \%$ confidence intervals. Right: Distribution of differences from expected healthy values among healthy subjects and patients with hydrocephalus. In this example, $\lambda_{1}$ of the $\mathrm{CR}$ is shown.

and 18 were included in the statistical analysis because the surgery in 1 patient had failed, resulting in no clinical or radiological improvement. Three DTI parameters reached statistical significance in this analysis: $\mathrm{FA}_{\mathrm{CR}}, \lambda_{\mathrm{ICR}}, \lambda_{\mathrm{BCR}}$ (Fig. 4). The $\Delta \Delta \mathrm{FA}$ was 0.07 (95\% CI $0.03-0.11), \Delta \Delta \lambda_{1}$ was $0.07 \times 10^{-3} \mathrm{~mm}^{2} / \mathrm{sec}\left(95 \%\right.$ CI $0.01-0.13 \times 10^{-3} \mathrm{~mm}^{2} /$ sec), and $\Delta \Delta \lambda_{3}$ was $-0.06 \times 10^{-3} \mathrm{~mm}^{2} / \mathrm{sec}(95 \% \mathrm{CI}-0.11$ to $-0.01 \times 10^{-3} \mathrm{~mm}^{2} / \mathrm{sec}$ ).

A comparison was made between the subgroup of patients treated with ETV (10 patients) and the subgroup treated with other surgical interventions ( 8 patients). The difference between the differences $(\Delta \Delta)$ for the non-ETV group reached statistical significance in 4 parameters: $\mathrm{FA}_{\mathrm{CR}}, \lambda_{3 \mathrm{CR}}, \mathrm{FA}_{\mathrm{GENU}}, \lambda_{3 \mathrm{GENU}}$. In the $\mathrm{CR}, \Delta \Delta \mathrm{FA}$ was 0.11 (95\% CI $0.06-0.16)$ and $\Delta \Delta \lambda_{3}$ was $-0.08 \times 10^{-3} \mathrm{~mm}^{2} / \mathrm{sec}$ ( $95 \% \mathrm{CI}-0.13$ to $-0.03 \times 10^{-3} \mathrm{~mm}^{2} / \mathrm{sec}$ ). In the GENU, $\Delta \Delta$ FA was -0.09 (95\% CI -0.14 to -0.04$)$ and $\Delta \Delta \lambda_{3}$ was $0.10 \times 10^{-3} \mathrm{~mm}^{2} / \mathrm{sec}\left(95 \%\right.$ CI $\left.0.01-0.19 \times 10^{-3} \mathrm{~mm}^{2} / \mathrm{sec}\right)$. This difference between the differences $(\Delta \Delta)$ for the ETV group showed the same trend of changes as the non-ETV group, yet those did not reach statistical significance.

\section{Discussion}

This large-cohort study, designed with a multidisciplinary approach combining clinico-neurological, radio- logical, and multiple DTI parameters, provides a comprehensive view of the benefits of using DTI for characterizing hydrocephalus. This work followed our initial experience with the changes in DTI parameters in hydrocephalus, which was presented in a case-control study of 7 patients with hydrocephalus. ${ }^{2}$ The present study reveals the most sensitive DTI parameters for hydrocephalus and suggests that they, in addition to conventional radiological parameters, can serve as a tool for the quantitative radiological assessment of hydrocephalus.

\section{Diffusion Tensor Imaging Characterization of Hydrocephalus}

The first aim in this study was to determine the most sensitive DTI parameters to identify and characterize patients with active hydrocephalus. We also examined the relationship between those parameters and the patients' clinical and radiological presentation.

Since DTI parameters vary with age, we had to standardize our data. We used our healthy subject group to plot trend lines for age-related expected DTI parameters. Our calculations were based on differences between the measured DTI parameters in the patients with hydrocephalus and the healthy trend lines. This standardization enabled accurate estimation of the DTI deviations at different ages.

We found that $\lambda_{1}$ of the CR was the most sensitive pa-

TABLE 5. Average differences between measured DTI parameters and the healthy values expected, and calculated sensitivity in identifying hydrocephalus

\begin{tabular}{|c|c|c|c|c|c|c|c|c|c|c|c|c|}
\hline \multirow[b]{2}{*}{ Parameter } & \multicolumn{4}{|c|}{ CR } & \multicolumn{4}{|c|}{ GENU } & \multicolumn{4}{|c|}{ OR } \\
\hline & FA & MD & $\lambda_{1}$ & $\lambda_{3}$ & FA & MD & $\lambda_{1}$ & $\lambda_{3}$ & FA & MD & $\lambda_{4}$ & $\lambda_{3}$ \\
\hline Average difference (D) & 0.134 & 0.084 & 0.315 & -0.032 & -0.130 & 0.266 & 0.161 & 0.274 & -0.030 & 0.230 & 0.240 & 0.182 \\
\hline Lower limit of $95 \% \mathrm{Cl}$ & 0.109 & 0.056 & 0.269 & -0.059 & -0.171 & 0.187 & 0.085 & 0.190 & -0.053 & 0.156 & 0.150 & 0.111 \\
\hline Upper limit of $95 \% \mathrm{Cl}$ & 0.159 & 0.112 & 0.361 & -0.005 & -0.089 & 0.344 & 0.236 & 0.358 & -0.008 & 0.303 & 0.329 & 0.253 \\
\hline Sensitivity for $95 \%$ specificity & $81 \%$ & $67 \%$ & $89 \%$ & $47 \%$ & $58 \%$ & $64 \%$ & $31 \%$ & $64 \%$ & $37 \%$ & $71 \%$ & $66 \%$ & $63 \%$ \\
\hline
\end{tabular}




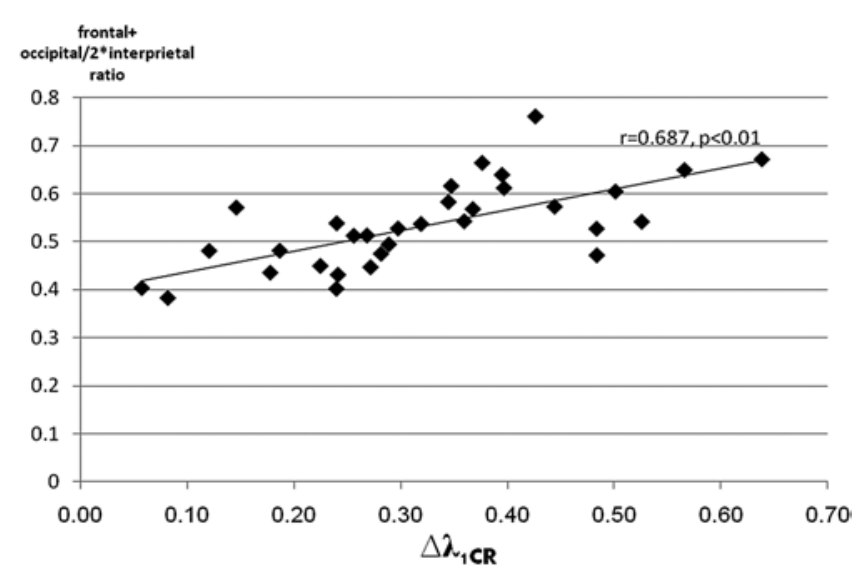

FIG. 3. Correlation between $\Delta \lambda_{\mathrm{ICR}}$ and the average of the frontal and occipital horn width divided by the interparietal distance. $r=0.687, p<$ 0.01 .

rameter for hydrocephalus (sensitivity $89 \%$ for $95 \%$ specificity).

We found that compared with normal controls, patients with active hydrocephalus had a statistically significant increase in FA, MD, and $\lambda_{1}$ and a decrease in $\lambda_{3}$ for the CR. In the GENU and OR there was a statistically significant decrease in FA and an increase in MD, $\lambda_{1}$, and $\lambda_{3}$. Several other studies have looked at DTI changes in patients with hydrocephalus and showed results with the same trend as in our study in the regions of the corpus callosum and the CR. ${ }^{1,2,6,9,15}$

In the CR, an increase in the FA and $\lambda_{1}$ and a decrease in $\lambda_{3}$ could reflect compression of the white fibers by fluid pressure in the ventricles, thus resulting in increased diffusion in the parallel axis of the fibers. ${ }^{2}$ An increase in MD in the CR may have several explanations: it could be a result of the degeneration of fibers, as happens several weeks after stroke, although the reversibility of some of the DTI parameters after surgery does not support this theory. Instead, it is more likely that the MD increase is

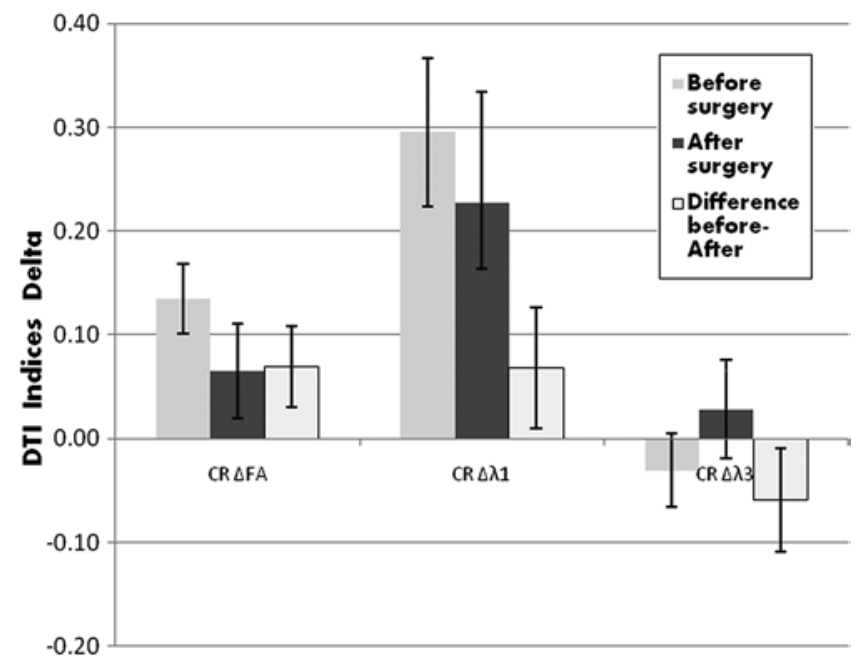

FIG. 4. Averages of differences from expected healthy values for DTI parameters of the CR with the $95 \%$ confidence intervals. caused by periventricular edema or that it merely represents a greater increase in $\lambda_{1}$ than the decrease in $\lambda_{3}$ that would result in total MD decline. In the GENU and OR, a decrease in FA and an increase in MD and $\lambda_{3}$ is a pattern that fits conditions such as stroke and demyelinating diseases. ${ }^{2,13,14}$ This pattern suggests that fibers in these areas undergo degeneration, a finding also presented pathologically. ${ }^{5}$ Another possible explanation for the change in the parameters in these areas is technical: while these fiber tracts in a healthy subject are wide, in a patient with hydrocephalus there is narrowing of the fiber tracts and a larger percentage of the voxels contain, beside the tract's fiber, additional tissues (like CSF) with higher diffusion, resulting in a partial volume artifact.

\section{Correlation of Clinico-Neurological, Radiological, and DTI Parameters}

To assess the potential clinical utility of DTI parameters, we compared the DTI values with clinico-neurological hydrocephalus severity grades as well as with qualitative conventional radiological parameters. We could not find a meaningful difference in the DTI parameters in the various disease severity groups except for $\lambda_{1}$ for the corpus callosum, which was statistically different in the severe hydrocephalus group as compared with that in the combined mild and moderate hydrocephalus subgroup. This result may indicate a true difference or may be the random result of multiple comparisons. Further studies with larger cohorts of control and hydrocephalus patients should be performed to validate this observation beyond the multiple comparison corrections. In a multiple regression analysis, the use of DTI parameters in addition to conventional radiological parameters did not provide additional benefit for assessing the clinical severity of hydrocephalus. Radiologically, there was good correlation between the DTI parameters and measurements representing ventricular size. Although DTI does not appear to have benefits surpassing those of conventional radiology, it does provide quantitative and objective measures of the pathology. Another way of looking at it is that DTI can provide an objective measure in cases in which the conventional qualitative assessment is under question.

It is possible that in our study group, the lack of a correlation between disease severity and DTI parameters was attributable to the use of a clinical grade and not a formal invasive ICP measurement. Another problem is the heterogeneity of our HCP group: some of the patients with hydrocephalus suffered from an additional clinical illness or condition, such as tumor or prematurity. It was difficult to differentiate between a clinical status attributed solely to hydrocephalus and factors associated with other clinical situations. However, the finding that some radiological parameters, such as periventricular edema, overall radiological evaluation of ICP, and pituitary flattening, were statistically significantly correlated with the clinical disease severity suggests that clinical grading does represent a difference within the severity groups. Therefore, it appears that in this large-cohort, multidisciplinary study, conventional radiological parameters interpreted by experienced radiologists are superior to DTI parameters in assessing clinical disease severity by using an imaging modality. 


\section{Neurosurgical Procedures and DTI}

After a successful surgical intervention, there was a statistically significant decline in the FA and $\lambda_{1}$ in the CR, as in previous studies. $1,2,7,9$ These parameters showed a trend toward healthy subjects' values but did not reach normal values. The improvement in these DTI parameters indicates that the hydrocephalus effect on CR fibers is, at least partially, reversible. In the GENU and OR, there was no change in the parameters measured after surgery. These findings support the notion that these fibers undergo a degenerative and probably irreversible process, as shown in previous studies. ${ }^{5}$

After surgical intervention, the patient subgroup treated with a VPS showed greater changes in DTI parameters than the subgroup treated with ETV. In the non-ETV subgroup, there was a larger change in the ventricle size postsurgery than that in the ETV subgroup. Since there is a correlation between ventricular size and DTI parameters, it is likely that the difference between the ETV and nonETV subgroups can be explained by the change per se in ventricular size.

\section{Conclusions}

In this comprehensive multidisciplinary study of patients with hydrocephalus, we searched for a correlation among standardized DTI parameters and clinical disease severity and conventional radiological parameters. In our HCP group we found that some of the DTI parameters could be sensitive and specific for hydrocephalus recognition and could be used as part of the overall radiological evaluation of hydrocephalus. We found that the DTI parameters correlated well with ventricular volume estimates. However, in the patient cohort in the present study, there was no convincing correlation between clinical disease severity and DTI parameters. Although our results indicate that DTI is beneficial for a radiological diagnosis of hydrocephalus, the ability of this imaging method to indicate disease severity should be evaluated in a larger cohort.

\section{References}

1. Air EL, Yuan W, Holland SK, Jones BV, Bierbrauer K, Altaye M, et al: Longitudinal comparison of pre- and postoperative diffusion tensor imaging parameters in young children with hydrocephalus. J Neurosurg Pediatr 5:385-391, 2010

2. Assaf Y, Ben-Sira L, Constantini S, Chang LC, Beni-Adani L: Diffusion tensor imaging in hydrocephalus: initial experience. AJNR Am J Neuroradiol 27:1717-1724, 2006

3. Basser PJ, Mattiello J, LeBihan D: MR diffusion tensor spectroscopy and imaging. Biophys J 66:259-267, 1994

4. Basser PJ, Pierpaoli C: A simplified method to measure the diffusion tensor from seven MR images. Magn Reson Med 39:928-934, 1998
5. Del Bigio MR, Wilson MJ, Enno T: Chronic hydrocephalus in rats and humans: white matter loss and behavior changes. Ann Neurol 53:337-346, 2003

6. Hattingen E, Jurcoane A, Melber J, Blasel S, Zanella FE, Neumann-Haefelin T, et al: Diffusion tensor imaging in patients with adult chronic idiopathic hydrocephalus. Neurosurgery 66:917-924, 2010

7. Leliefeld PH, Gooskens RH, Braun KP, Ramos LM, Uiterwaal CS, Regli LP, et al: Longitudinal diffusion-weighted imaging in infants with hydrocephalus: decrease in tissue water diffusion after cerebrospinal fluid diversion. J Neurosurg Pediatr 4:56-63, 2009

8. O'Hayon BB, Drake JM, Ossip MG, Tuli S, Clarke M: Frontal and occipital horn ratio: A linear estimate of ventricular size for multiple imaging modalities in pediatric hydrocephalus. Pediatr Neurosurg 29:245-249, 1998

9. Osuka S, Matsushita A, Yamamoto T, Saotome K, Isobe T, Nagatomo Y, et al: Evaluation of ventriculomegaly using diffusion tensor imaging: correlations with chronic hydrocephalus and atrophy. J Neurosurg 112:832-839, 2010

10. Pajevic S, Pierpaoli C: Color schemes to represent the orientation of anisotropic tissues from diffusion tensor data: application to white matter fiber tract mapping in the human brain. Magn Reson Med 42:526-540, 1999

11. Pierpaoli C, Basser PJ: Toward a quantitative assessment of diffusion anisotropy. Magn Reson Med 36:893-906, 1996

12. Pierpaoli C, Jezzard P, Basser PJ, Barnett A, Di Chiro G: Diffusion tensor MR imaging of the human brain. Radiology 201:637-648, 1996

13. Song SK, Yoshino J, Le TQ, Lin SJ, Sun SW, Cross AH, et al: Demyelination increases radial diffusivity in corpus callosum of mouse brain. Neuroimage 26:132-140, 2005

14. Warach S, Gaa J, Siewert B, Wielopolski P, Edelman RR: Acute human stroke studied by whole brain echo planar diffusion-weighted magnetic resonance imaging. Ann Neurol 37:231-241, 1995

15. Yuan W, Mangano FT, Air EL, Holland SK, Jones BV, Altaye $\mathrm{M}$, et al: Anisotropic diffusion properties in infants with hydrocephalus: a diffusion tensor imaging study. AJNR Am J Neuroradiol 30:1792-1798, 2009

\section{Author Contributions \\ Conception and design: Ben-Sira, Assaf, Bassan, Constantini. Acquisition of data: Ben-Sira. Analysis and interpretation of data: Goder, Bassan, Lifshits. Drafting the article: Ben-Sira, Goder. Critically revising the article: Ben-Sira, Constantini. Reviewed submitted version of manuscript: Assaf. Approved the final ver- sion of the manuscript on behalf of all authors: Assaf. Statistical analysis: Goder. Administrative/technical/material support: Bas- san. Study supervision: Constantini.}

\section{Correspondence}

Liat Ben-Sira, Department of Pediatric Neurosurgery, Tel Aviv Sourasky Medical Center, 6 Weizmann St., Tel Aviv 64239, Israel. email: bensiraliat@gmail.com. 